\title{
An Assessment of Probabilistic Reactive Routing Protocol for MANET's
}

\author{
Aditya Pare \\ M-Tech Scholer \\ Department of Computer Science \\ RKDF Institute Bhopal
}

\author{
Gaurav Shrivastav \\ Assistant Professor \\ Department of Computer Science \\ RKDF Institute Bhopal
}

\begin{abstract}
MANET is an temporary decentralized infrastructure less network where each node can behave like receiver and transmitter and having a permission to move any were across the network without loss the connectivity with rest of network. Message sharing is an important task of MANET, MANET have verity of routing protocol to serve massage sharing .Performance of these routing protocol depend upon collision rate ,reach ability, rebroadcasting and average latency. Message sharing scheme in MANET is categorized namely into deterministic and probabilistic schemes. This paper give an bird eye over MANET and its routing algorithms mainly probabilistic scheme for routing and also include recent research towards better performance in term of lower collision rate, battery consumption, rebroadcasting rate and higher degree of reach ability, average latency.
\end{abstract}

\section{Keywords}

MANET, Probabilistic Algorithm, Bayesian Approach

\section{INTRODUCTION}

With incredible expansion of uses of personal computers and mobile computers lead to need a wireless network for sharing the information among the different computers. Wireless networks come in picture in mid of 1970 s and their interest has been growing gradually. But during the last two decade, because of internet (also known as global information sharing system) importance of Wireless network has been exploded [3]. But at that time information sharing between mobile node in limited resource and without uses of any centralized administration is difficult.But now this day a temporary infrastructure less wireless network having no central access point or any centralized administration is in existence and known as Mobile Ad-Hoc Network (MANET) [1]. Mobile ad-hoc networks encompass number of quality, use dynamic network topology, having limited bandwidth and consume less battery power. Mobile ad hoc network is significant for military operation to provide communication between squads, emergency case in out-of-the-way places, medical control etc. Whereas higher degree of node mobility lead to changes network topology because of that routing is a challenging task in ad-hoc network ever since it's came into existence [2].

This paper gives a descriptive overview regarding ad-hoc network and their routing protocol especially reactive routing protocol along with probabilistic routing algorithm, Bayesian probabilistic routing algorithm and value of affinity index, mutual affinity index and other parameter for probabilistic routing algorithms.

The presented paper concludes in total seven sections including this one. The second section gives an idea of mobile ad-hoc network, third section describe different routing protocol and their hierarchy. The fourth section illustrates probabilistic routing approach over MANET. Fifth section throws some light on previous work of MANET. Finally the paper concludes in six section and reference in seventh section.

\section{MOBILE AD-HOC NETWORK}

Mobile ad-hoc network is temporary infra structure less computer network where node of network can be move any were within the network without any centralized authority. MANET initially introduce and used by military in the field of war and at the time of natural digesters in sort duration of time with limited resource in order to establish communication channel. Each node in MANET works as independent router having both transmitter and receiver and communicate directly with other node that's comes under its radio range (radio range is generally transmitting and receiving range of any node) as show in figure 1 number with dark circle is an independent node whereas dashes circle represent radio range of respective node $[1,4]$

MANET is also known as Cooperative ad-hoc networks Communication over the mobile ad-hoc network is performed by stations cooperation with each other, i.e., if any node want to communicate with other node that's not comes under its radio range then it is the responsibility of intermediate node also know as relay node to maintain communication between source and destination.

MANET is very advantageous to use provide services and sharing the information regardless of geographical position, time and resources. Because of above special feature mobile ad-hoc network windy used in Military operation, rescue operation by disaster management team ,police exercises in remote aria ,Sensor networks, VNET, PAN for communication of several portable devices etc [5].

Along with above encouraging points there are some limitations in MANET. MANET has higher degree of security threads, lack of authorization facilities and Volatile network topology makes its very hard to identify misbehave nodes due to volatile network topology $[4,5]$. 


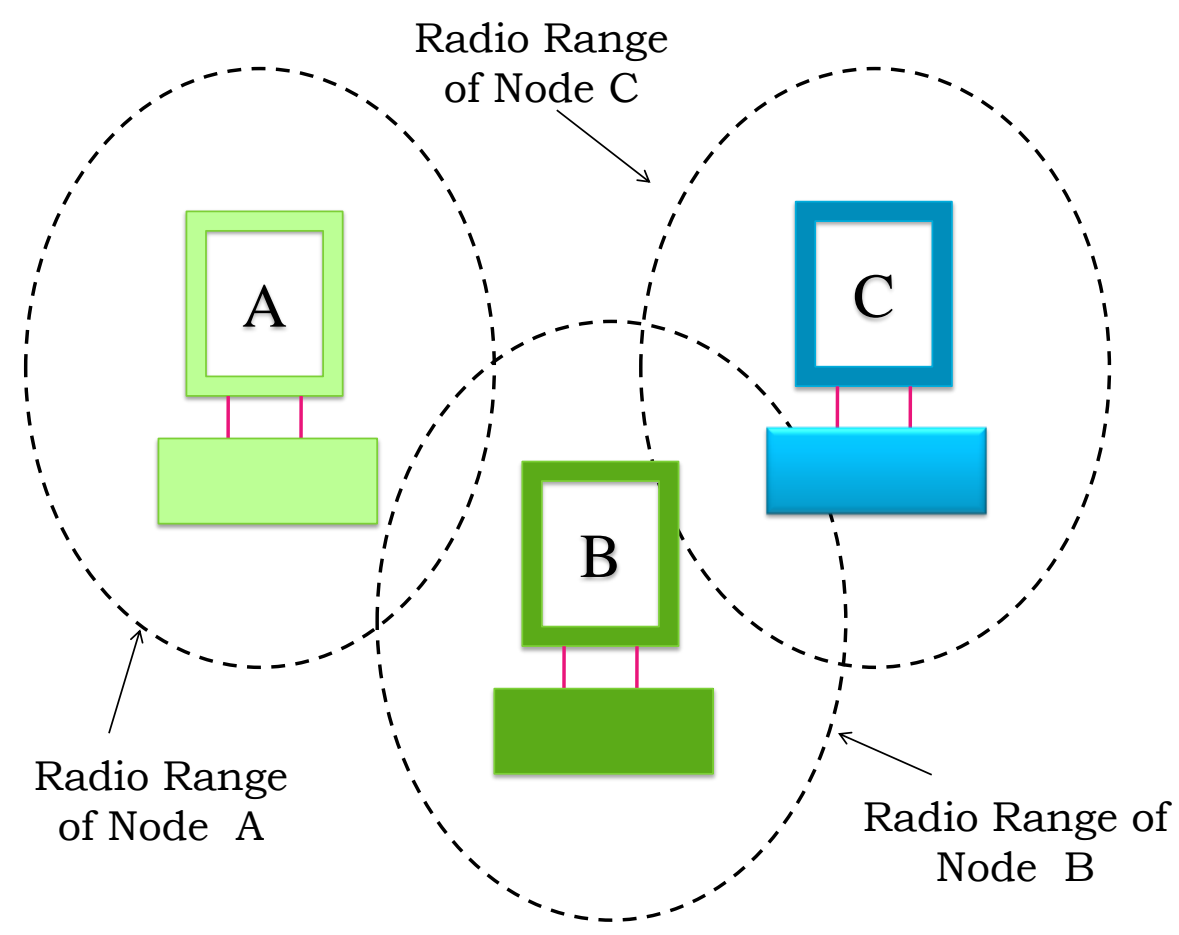

Figure 1 Scenario of MANET

\section{PROTOCOLS USED IN MANET}

Routing protocol used in MANET for establishment of communication over network between mobile nodes is namely divided into three types proactive, reactive and hybrid. Figure 2 shows the hierarchy of the protocols.

1. Proactive protocol: - Proactive MANET routing protocol (PMPs) is a table driven routing protocol, need to continuously update network topology information and guarantee that it is presented to all nodes. PMPs lead to reduce time delay of packet over the network but simultaneously increase data overhead by continuously updating routing information. DSDV, WRP, CGSR are the example of proactive protocols.

2. Reactive MANET protocols:-Reactive MANET protocols is an on demand routing protocol, need to path hunting only when there is need of the path. Lower protocol data over head is advantage with Reactive protocol but having longer packet delays. There is number of reactive protocol like AODV, DSR and TORA.
3. Hybrid MANET routing protocols:-The Hybrid MANET routing protocols is an advantageous amalgamation of both proactive and reactive MANET protocols with better resultant protocol overhead and packet delay than any of individual protocols[6]. It is also capable of regulating packet dynamically on the basis of different network conditions. ZRP protocol is an example of hybrid model.

This paper gives a main concentration over probabilistic approach of routing and present different flavor of probabilistic reactive routing protocol as suggest in recent research in related work section of this paper.

Some of basic reactive routing protocols are describe below.

DSR: DSR routing protocol is used to suggest Multiple routes and for Promiscuous overhearing but its suffer from scalability problem and large delay.

AODV: AODV is highly applicable for dynamic topology but it also suffer form scalability and larger delay problem along with that AODV having higher degree of protocol overhead. 


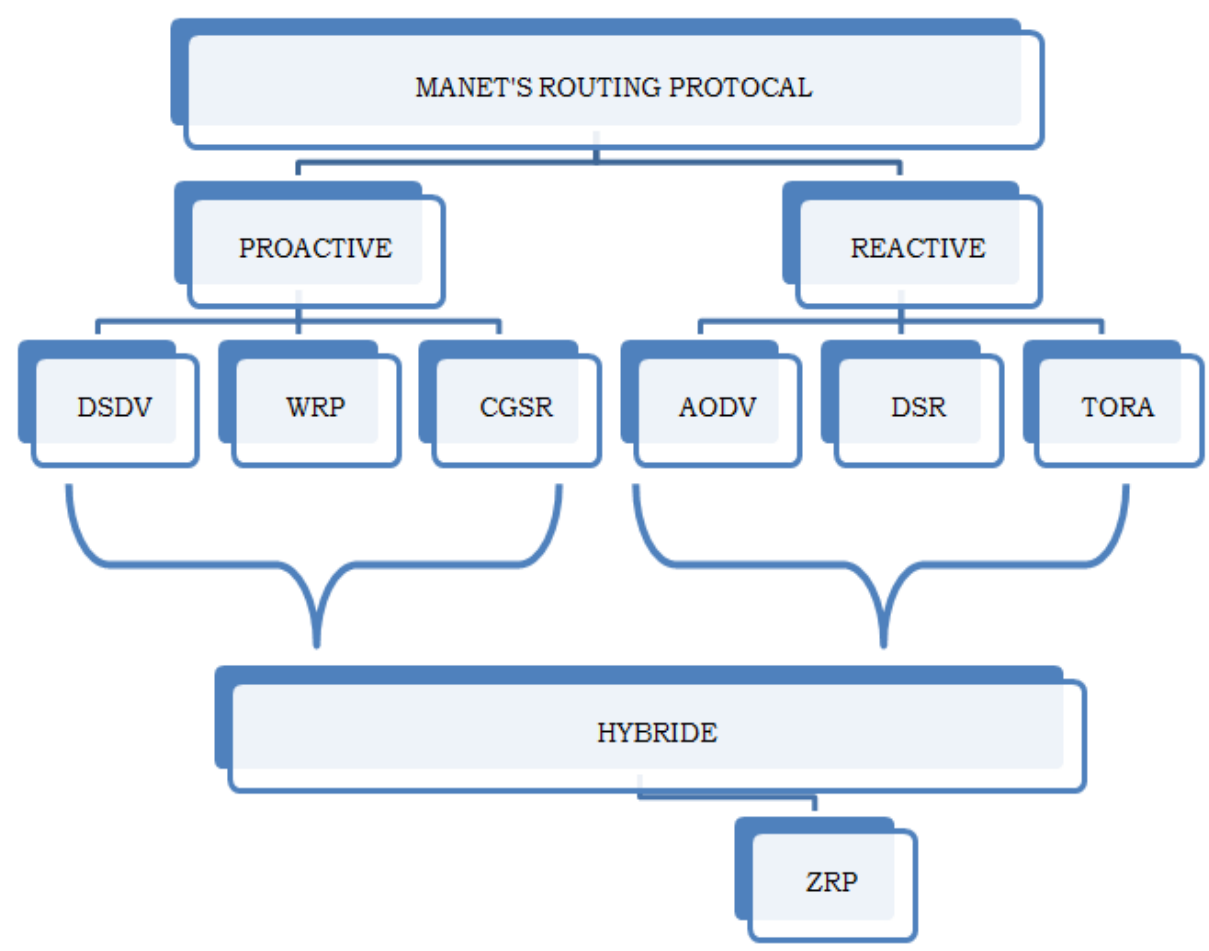

Figure 2 Hierarchy of MANET Routing Protocol

In recent research AODV is might to be updated and gives AODV-BR, AOMDV[18], AODV-ABR[19].Where AODV-BR having higher throughput than AODV but even efficient for frequently changing network. AOMDV lead to minimized routing overhead and coordination overhead by intermediate node but performance of AOMDV going to degrade in moderate and sparse network. AODV-ABR is more appreciable in higher dynamic network with lower control overhead but having lower probability to find alternate route.

TORA:- TORA provides the reliable supports for sensing link status towards neighbor node that's comes under its radio range. TORA supports higher degree of security authentication and lower degree of control overhead [17, 18]. It depends on synchronized clocks among nodes in the ad hoc network \& dependence over intermediate lower layers for certain functionality presumes with higher overhead[17].

\section{PROBABILISTIC ALGORITHM}

In probabilistic routing algorithms for MANET calculation for route suggestion between any sources to destination end is depend upon probability of past lower rebroadcasting rate. Probabilistic approach tries to minimized rebroadcast. In a probabilistic scheme, nodes transmit a message with a pre determined probability $\mathrm{p}$.

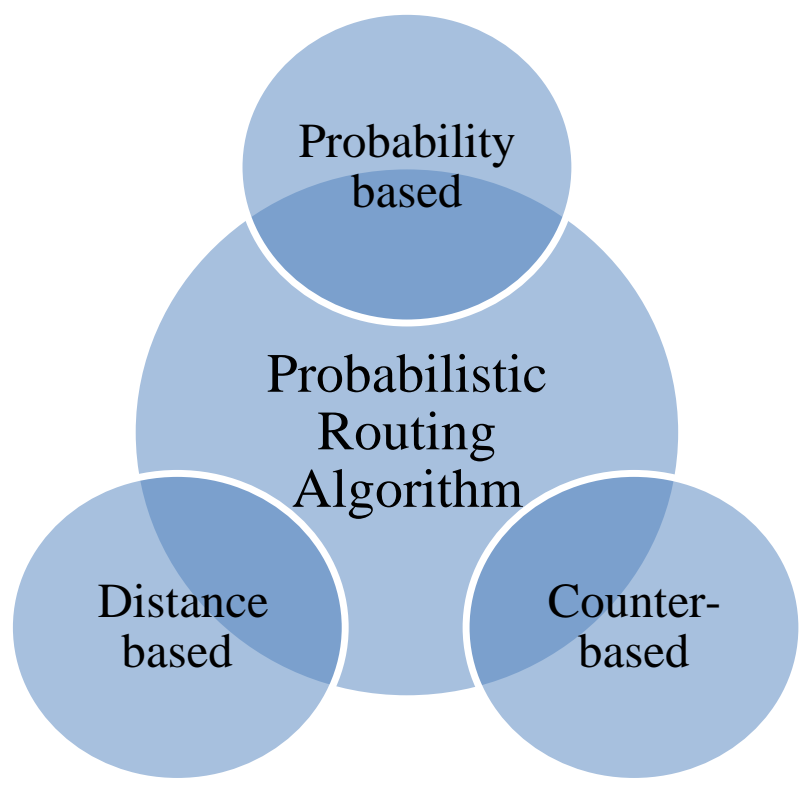

Figure 3:- Classification of Probabilistic routing algorithms

Recent research shows that rebroadcasting rate in probabilistic approach is significantly lower as compared to blind flooding in deterministic routing approach. Probabilistic schemes that's had 
been proposed in past comes under three categories namely probability-based, counter-based and distance-based.

Each probability model is represented by the equation $[5,6,7]$ :

$$
P^{n}=f\left(s, r_{n}, p\right)
$$

Where Pn is the probability of successful transmission via radio node ( $\mathrm{rn}$ ) from source node (s) that comes under the radio range of source node ( $\mathrm{s}$ ). The function $\mathrm{f}$ depends on the specific protocol being analyzed.

Bayesian approach is one of most popular probabilistic method that's works on the basis of historical record of successful transmission via any intermediate node. Figure 3 shows the basic functionality of the Bayesian Approach.

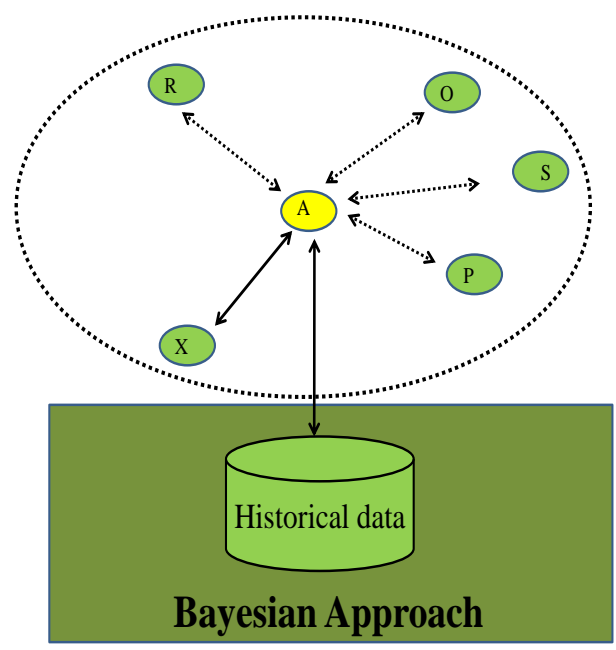

Figure 3 Bayesian Approach

Bayesian probabilistic approach depend upon singular affinity index (SAI) ie any node want to transmits any packet towards destination then source node (A) apply Bayesian probability over all of its radio node and find most probabilistic node on the basis of historical data and send packet via that selected node. As show in figure 3 solid arrow line show selected node for transmission. But degree of accuracy in case of singular affinity index is not up to mark; mostly during the initiation of network probability of rebroadcast is very high.

Where some enhancement version of Bayesian approach used mutual affinity index to calculate probability of any successive node for packet forwarding which is depend upon belief function, procedure of calculation belief function is show in equation $1[9,10]$.

$$
\begin{gathered}
\operatorname{Bel}(A)=\sum_{B / B \in A} m(b) \ldots 1 \\
\mathrm{M}_{\mathrm{ai}}\left(\frac{\mathrm{w}_{\mathrm{i}}}{\mathrm{l}_{\mathrm{j}}}\right)=\pi_{\mathrm{j}=1}^{\mathrm{m}}\left[\mathrm{l}_{\mathrm{ij}_{1}}+\mathrm{l}_{\mathrm{ij}_{2}}+\mathrm{l}_{\mathrm{ij}_{3}}+\cdots+\mathrm{l}_{\mathrm{ij}_{\mathrm{k}}}\right] / \mathrm{n}_{\mathrm{i}}
\end{gathered}
$$

$$
\mathrm{M}_{\mathrm{ai}}=\pi_{\mathrm{j}=1}^{\mathrm{m}}\left\lceil\frac{\sum_{\mathrm{r}=1}^{\mathrm{k}} \mathrm{w}_{\mathrm{ijr}}}{\mathrm{n}_{\mathrm{i}}}\right\rceil
$$

Now since we are multiplying the probabilities of each and every attribute hence; even if one of the attributes has a zero probability; the whole index will become zero. Because of this; zero probability will be replaced with a very low probability (0.001).

\section{PREVIOUS WORK}

Many research works has been done in this area. Some of them are presented here.

The author presents and shows obtained results in his research. This work is based on Bayesian approach, Queuing theory and reliability theory. Here the methods bound of application of Bayesian approach to some problems of queuing theory and reliability theory. This approach could be used, for instance, for calculating moment and quintile characteristics for performance and reliability characteristics of large groups of systems or devices [11].

Nadia Bali [12] proposed a hierarchical Markov model for the sources with a common hidden classification field which is modeled via a Potts-Markov field. Classical methods of dimensionality reduction in hyper spectral imaging use classification methods either to classify the spectra or to classify the images in classes where is, in general, much less than the number of spectra or the number of observed images. However, these methods neglect either the spatial organization of the spectra or the spectral property of the pixels along the spectral bands.

The processes for decision making problems based on the use of the Dempster-Shafer (D-S) theory can be able in different ways according to the requirements of each single problem. In this input the authors present a decision making scheme based on Dempster-Shafer (D-S) theory. Jose M. Merigo [13] proposed the use of a hybrid averaging operator (2-THA) which uses the 2-tuple linguistic representation model. Due to use of 2-THA in D-S theory, obtained a new aggregation operator: the belief structure - 2-THA (BS-2-THA) operator and studied some of its main properties and then show the descriptive example of the new approach in a decision making problem.

Nidhi S Kulkarni [14] study and analyses the on demand routing protocols in deep and gives an overview of the existing on demand routing protocols and a parametric comparison is made with the recently developed protocols, also covers some important conventional routing protocols and the recently proposed extensions of AODV. These protocols are the multipath extensions of Ad Hoc On Demand Distance Vector routing protocol (AODV) such as AODV with break avoidance (AODV-BR), Scalable Multipath on demand routing (SMORT).

One of the typical routing methods in mobile ad-hoc networks use on demand distance vector, or Ad-hoc On-demand Distance Vector (AODV). The key concern in this protocol is the cost of route establishment. Rusheel Jain [15] suggested an efficient routing algorithm for mobile ad-hoc networks with a route establishment technique using Bayesian approach. They consider both time and space information to compute the route from source to destination. The results show that there is major 
improvement in delivery ratio, control packets overhead w.r.t. mobility and control packet overhead w.r.t. network size .

\section{CONCLUSION}

This paper gives a bird eye over routing algorithm in MANET. Routing algorithm generally classify into two subtype deterministic and probabilistic routing algorithms. Deterministic routing algorithm suffer from blind flooding whereas probabilistic routing algorithm overcome the problem of blind flooding and maintain historical record of time and space for all node to maintaining network traffic in order to reduce probability of collision, rebroadcast at the expense of reach ability and enhanced scheme has higher throughput, lower latency and better reach ability.

\section{REFERENCES}

[1]. B. Williams and T. Camp, Comparison of broadcast in techniques for mobile ad hoc networks. In Proc. ACM Symposium on Mobile Ad Hoc Networking \& Computing (MOBIHOC 2002), pp. 194-205, 2002.

[2]. S.-Y. Ni, Y.-C. Tseng, Y.-S. Chen, and J.-P. Sheu, "The broadcast storm problem in a mobile ad hoc network", Proc.Mobicom_99, 1999.

[3]. Y. Sasson, D. Cavin, and A. Schiper, Probabilistic Broadcast for flooding in wireless mobile ad hoc networks, In Proc. IEEE Wireless Communications \& Networking Conference (WCNC 2003), pp. 1124-1130, March 2003.

[4]. J. Wu and W. Lou, "Forward-node-set-based broadcast in clustered mobile ad hoc networks," Wireless Communication and Mobile Computing, vol. 3, pp. 155 173, 2003.

[5]. A. Keshavarz-Haddad, V. Ribeiro, and R. Riedi, "ColorBased Broadcasting for Ad Hoc Networks," in Proceeding of the 4th International Symposium on Modeling and Optimization in Mobile, Ad Hoc, and Wireless Network

[6]. (WIOPT" 06). Boston, MA, 2006, pp. 1 - 10.

[7]. J. Cartigny and D. Simplot, "Border node retransmission based probabilistic broadcast protocols in ad hoc networks," Telecommunication Systems,, vol. 22, pp. 189204, 2003.

[8]. C. E. Perkins, and E. M. Royer, "Ad-hoc on-demand distance vector routing," 2nd IEEE Workshop on Mobile Computing Systems and Applications, Monterey, California, USA: Feb 25 - 26, 1999: 90-100.

[9]. H D-Ferriere, M Grossglauser, and M Vetterli, "Age Matters: Efficient Route Discovery in Mobile Ad Hoc
Networks Using Encounter Ages," 4th ACM International Symposium on MANET and Computing, 2003

[10].C. E. Perkins, E. M. Belding-Royer, and S. Das, “Ad hoc on-demand distance vector (AODV) Routing," RFC 3561, July 2003,

[11].David Johnson, David Maltz and Yih-Chun Hu, "The Dynamic Source Routing Protocol for Mobile Ad Hoc Networks," Internet Draft, draft-ietf-manet-dsr10.txt,July 2004

[12]. Alexey Kudryavtsev and Sergey Shorgin, "On the Bayesian Approach to the Analysis of Queueing Systems and Reliability Characteristics", IEEE 2010, pp 1042-1045.

[13].Nadia Bali and Ali Mohammad-Djafari, "Bayesian Approach With Hidden Markov Modeling and Mean Field Approximation for Hyperspectral Data Analysis", IEEE 2008, pp 217-225.

[14].Jose M. Merigo, Montserrat Casanovas and Luis Martinez, "A Decision Making Model Based on Dempster-Shafer Theory and Linguistic Hybrid Aggregation Operators", IEEE 2008, pp 180-185.

[15].Nidhi S Kulkarni, Balasubramanian Ramant, and Indra Gupta, "On Demand Routing Protocols for Mobile Ad Hoc Networks: A Review”, IEEE 2009, pp 586-591.

[16].Rusheel Jain, Murali Parameswaran and Chittaranjan Hota, "An Efficient On-Demand Routing Protocol for MANETs using Bayesian Approach", IEEE 2011

[17].Mehran Abolhasan, Tadeusz Wysocki, Eryk Dutkiewicz,“A review of routing protocols for mobile ad hoc networks" in Elsevier,2004

[18].TamilarasanSanthamurthy,“A Quantitative Study and Comparison of AODV, OLSR and TORA Routing Protocols in MANET" in International Journal of Computer Science Issues, Vol. 9, Issue 1, No 1, January 2012

[19].Sunil Taneja and Ashwani Kush"A Survey of Routing Protocols in Mobile Ad Hoc Networks" in International Journal of Innovation, Management and Technology, Vol. 1, No. 3, August 2010 ,ISSN: 2010-0248

[20].Pankaj Palta and Sonia Goyal,"Comparison of OLSR and TORA Routing Protocols Using OPNET Modeler" in International Journal of Engineering Research \& Technology (IJERT),Vol. 1 Issue 5, July - 2012,ISSN: 2278-01 